\title{
The clinical use of serum Mas-Related G Protein-Coupled Receptor-X2 as a diagnostic biomarker is questionable
}

\author{
Grzegorz Porebski ${ }^{1}$, Alicja Dziadowiec ${ }^{2}$, and Mateusz Kwitniewski \\ ${ }^{1}$ Uniwersytet Jagiellonski w Krakowie Collegium Medicum \\ ${ }^{2}$ Uniwersytet Jagiellonski w Krakowie Wydzial Biochemii Biofizyki i Biotechnologii
}

June 29, 2021

Title: The clinical use of serum Mas-Related G Protein-Coupled Receptor-X2 as a diagnostic biomarker is questionable

Authors: Grzegorz Porebski (1), Alicja Dziadowiec (2), Mateusz Kwitniewski (2)

Affiliations: (1) Department of Clinical and Environmental Allergology, Jagiellonian University Medical College, Krakow; (2) Department of Immunology, Faculty of Biochemistry, Biophysics and Biotechnology, Jagiellonian University, Krakow

To the Editor,

Mas-related G protein-coupled receptor-X2 (MRGPRX2) is mainly expressed in the skin mast cells (MCs) and in the sensory neurons. ${ }^{1,2}$ It's activated by basic neuropeptides (e.g. Substance P, vasoactive intestinal peptide), by antimicrobial host defense peptides, by eosinophil granule proteins, or by a number of commonly used small-molecule drugs (e.g. neuromuscular blocking agents, fluoroquinolones, vancomycin). ${ }^{1-4} \mathrm{MCs}$ are key effector cells in a number of diseases, and MRGPRX2 has been considered to play an important role in non-IgE-mediated degranulation of MCs. Therefore, the contribution of MRGPRX2 in allergic reactions in mastocytosis and in mast cell activation syndromes, in those for instance induced by hymenoptera venom, has been postulated. ${ }^{1}$ The expression of MRGPRX2 has been shown to be up-regulated in skin-derived MCs in patients with severe chronic urticaria. ${ }^{4}$ Furthermore, research carried out by An and colleagues have demonstrated that MRGPRX2 is present in the serum of healthy individuals (12.9 ng/mL), that MRGPRX2 serum levels are elevated in nonallergic asthmatics $(79.8 \mathrm{ng} / \mathrm{mL})$, and that there is an even more elevated serum level in the subgroup of patients with allergic asthma $(102.4 \mathrm{ng} / \mathrm{mL})$. The receptor concentrations were also positively associated with a dose of inhaled corticosteroids and a level of asthma control. ${ }^{5}$ In conclusion, the authors of the research proposed that serum MRGPRX2 levels can be used as a potential independent biomarker for monitoring treatment outcomes in allergic asthma.

Based on the research findings presented above, we attempted to validate the utility of MRGPRX2 serum levels as a biomarker in a pilot group of patients presenting medical conditions of interest in comparison to healthy controls $(n=4)$. The patients' group involved: (a) three individuals under hymenoptera venom immunotherapy due to venom-triggered anaphylaxis, with the accompanying increased levels of serum tryptase (one of them with systemic mastocytosis); (b) three individuals with severe atopy confirmed by high levels of allergen-specific IgE to five or more common inhalant allergens, and who also were suffering from allergic asthma $(n=2)$ and chronic urticaria $(n=1)$. The detailed characteristic of analyzed subjects is summarized in the supplementary Table S1. MRGPRX2 serum levels were determined by ELISA (Abbexa) according to the manufacturer's protocol. Unexpectedly, we noted neither any significant increase in MRGPRX2 serum levels in patients, nor significant differences between subgroups (a) and (b), and between patients and controls. The recorded results remained in the range $0-0,49 \mathrm{ng} / \mathrm{ml}$ (Fig. 1A), and fluctuated around the detection limit 
of the ELISA. To clarify any discrepancies between our results and the data published by An, we reevaluated the same serum samples with the kit used by An and colleagues. ${ }^{5}$ (MyBioSource). We used human dermis and human dermal fibroblast homogenates as MRGPRX2 positive, and negative controls, respectively. The presence of MRGPRX2 transcript was determined by means of PCR (Fig. 2). In contrast to Abbexa, high serum MRGPRX2 levels were detected in all of the samples (Fig. 1B). Interestingly, there were no differences in MRGPRX2 levels between the positive and negative controls as well as between the patients' subgroups and the healthy controls. Moreover, in diluted samples, the receptor concentrations were higher (mean range $33-133 \mathrm{ng} / \mathrm{mL}$ ), which may indicate the presence of non-specific binding of detecting antibody. Details of laboratory methods are presented in the supplementary materials.

To conclude, our data suggest that MRGPRX2 serum levels measured by ELISA are not increased, neither in the healthy, nor in the patients with common disorders with mast cells involvement. This observation is in line with the findings of Ding et al. which showed that MRGPRX2 is present in whole blood, but not in human serum. ${ }^{3}$ In turn, the presence of MRGPRX2 in whole blood is supported by results reported recently by Wedi and colleagues on the receptor expression on peripheral blood basophils and eosinophils. ${ }^{2}$ Moreover, caution should be exercised while selecting ELISA kit targeting MRGPRX2 because of the potential risk of the occurrence of unspecific bindings since positive and negative control samples did not reveal any a differences when MyBioSource kit was used.

1. Porebski G, Kwiecien K, Pawica M, Kwitniewski M. Mas-Related G Protein-Coupled Receptor-X2 (MRGPRX2) in Drug Hypersensitivity Reactions. Front Immunol. 2018;9:3027.

2. Wedi B, Gehring M, Kapp A. The pseudoallergen receptor MRGPRX2 on peripheral blood basophils and eosinophils: Expression and function. Allergy. 2020;75:2229-2242.

3. Ding Y, Zhang T, Liu R, Che D, Wang N, He L. A novel ELISA method to determine human MrgX2 in chronic urticaria. Clin Transl Allergy. 2020;10:61.

4. Fujisawa D, Kashiwakura J, Kita H, et al. Expression of Mas-related gene X2 on mast cells is upregulated in the skin of patients with severe chronic urticaria. J Allergy Clin Immunol. 2014;134:622-633.e9.

5. An J, Lee JH, Won HK, et al. Clinical significance of serum MRGPRX2 as a new biomarker in allergic asthma. Allergy. 2020;75:959-962.
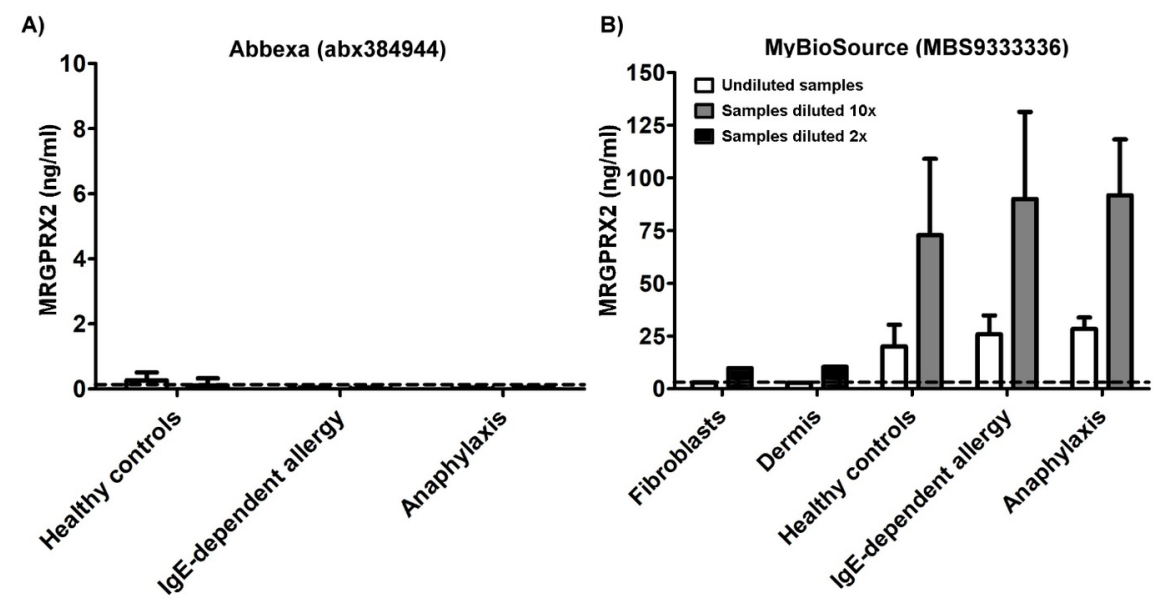

Figure 1 - MRGPRX2 serum levels in healthy controls and allergic patients were measured by Abbexa (A) or by MyBioSource (B) ELISA kits. The detection ranges of the Abbexa and the MyBioSource assays are $0.156 \mathrm{ng} / \mathrm{ml}-10 \mathrm{ng} / \mathrm{ml}$ and $3.12 \mathrm{ng} / \mathrm{ml}-100 \mathrm{ng} / \mathrm{ml}$, respectively. The dashed line indicates the detection limit. 


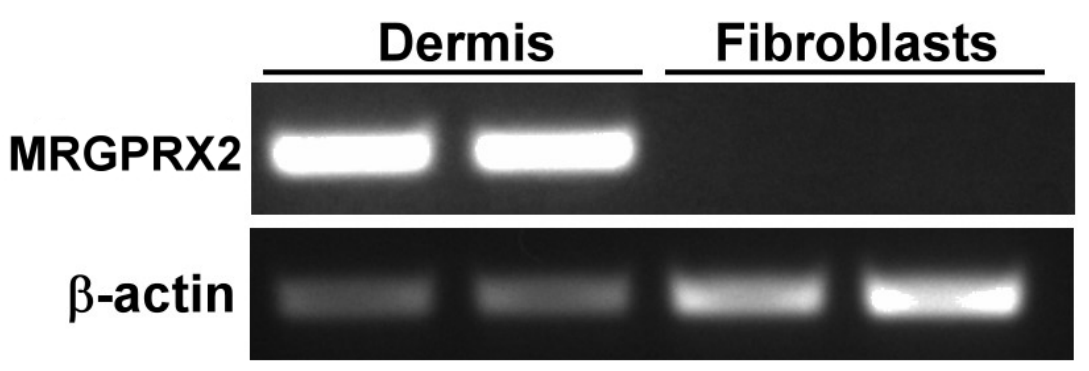

Figure 2 - Electrophoretic analysis of MRGPRX2 PCR products. RNA was isolated from both cultured human dermal fibroblasts and from whole human dermis. Afterwards, reverse-transcription PCR followed by PCR were performed. Beta actin was used as a positive control. 